\title{
Application of Real-time Communication Technology in Remote Monitoring System
}

\author{
Xia Ting ${ }^{1, a^{*}}$, Chen Yuting ${ }^{2, b}$ and Liu Chunyan ${ }^{1, c}$ \\ ${ }^{1}$ Wuhan University of Technology Huaxia College, Wuhan, China \\ ${ }^{2}$ China Ship Development and Design Center, Wuhan, China \\ a sunnyxting@qq.com, bytbob@gmail.com, $94394912 @ q q . c o m$
}

Keywords: remote monitoring; ZigBee; CGl; Socket; FLASH

\begin{abstract}
In order to realize remote monitoring, real-time communication technology must be applied. A wireless communication network was built with a plurality of sensors by ZigBee technology. Then data could be acquired and transmitted to the monitoring server wirelessly. The embedded microprocessor Cortex-A8 was used as the core of the monitoring server. It integrated ZigBee-Ethernet gateway, Web server and database. The remote communication model is B/S and $\mathrm{C} / \mathrm{S}$ collaborative model. CGI programs were designed to accomplish communication between browser and web server. Socket technology was applied to transmit signals between monitoring server and FLASH monitoring module in client. And then dynamic graphics could be realized by adopting FLASH technology. The tests show that Internet terminals can monitor the real-time status of equipment remotely and dynamic oscillogram of each signal is displayed without flicker.
\end{abstract}

\section{Introduction}

Since the Sate Council regarded the internet of things as strategic new industries in 2010, the researches of things of internet have become a hot issue gradually. Dr. Zhixi Fang the president of Intel China Research Institute said: "The high-profile internet of things technologies must be based on the development of embedded technologies, and the embedded system can improve the internet of things industry." So embedded technologies are the foundation and the key of the internet of things[1]. The remote monitoring system is a typical product of the internet of things. And real-time communication technology plays a vital role in the system. It enables a seamless connection between Internet and equipment.

\section{The Architecture of Remote Monitoring System}

Remote monitoring system can monitor the equipment status by browser, and warn when abnormities occur. The equipment performance can be evaluated by analyzing the historical status. According to the assessment results, the equipment can be repaired pertinently. The architecture of remote monitoring system is shown in Fig.1.

The monitoring system is designed according to the three layers of the Internet of things[2].

(1) Sensation Layer: It contains a plurality of sensors. A sensor and a ZigBee module constitute a wireless communication node. And many communication nodes form ZigBee wireless communication network. This Layer completes the data acquisition.

(2) Transmission Layer: The monitoring server integrates gateway, Web server and database. The high-performance 32-bit embedded processor Cortex-A8 is used as the core. This Layer completes the data communication.

(3) Application Layer: Monitoring stations can access monitoring server by Ethernet. Local Area Networks (LAN) are interconnected by switches and routers. Then terminals such as mobile users and internet users can monitor real-time data remotely. 


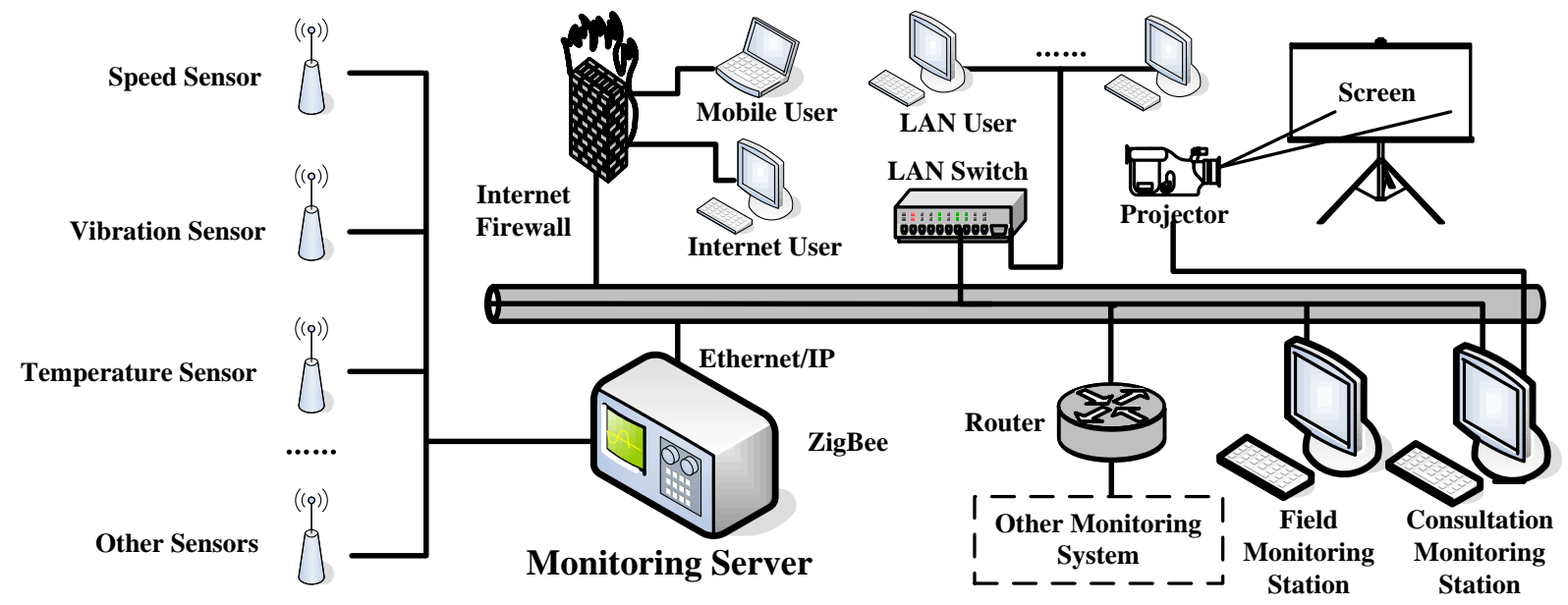

Fig.1. The architecture of remote monitoring system

\section{The Communication Mode of Remote Monitoring System}

The communication mode between remote monitoring system layers is shown in Fig.2.

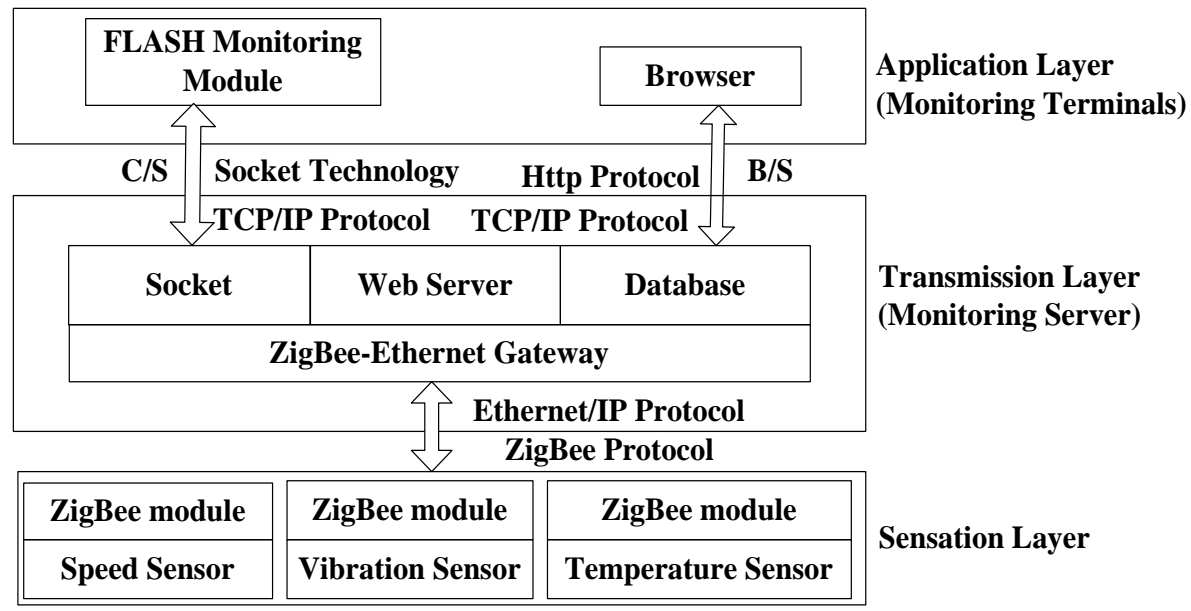

Fig.2. The communication mode of remote monitoring system

In order to connect the ZigBee wireless network to Internet, the ZigBee-Ethernet gateway is required. It is integrated in the monitoring server. By porting the EtherNet/IP (Ethernet Industrial Protocol) and zstack (ZigBee protocol stack) based on IEEE802.15.4, the gateway is compatible with the ZigBee network and connects with Ethernet and Internet seamlessly. C/S and B/S mode are adopted between transmission layer and application layer. C/S mode is used between monitoring server and FLASH monitoring module. Because C/S mode not only can ensure security and stability, but also can transmit large amounts of data frequently[3]. B/S mode doesn't have these characteristics. $\mathrm{B} / \mathrm{S}$ mode is used between monitoring server and FLASH monitoring module. A variety of scattered signals focus on a unified platform and terminals can get the equipment status quickly and easily[3].

\section{Real-time Communication Technology}

ZigBee Technology. Radio frequency chip CC2530 is adopted to realize ZigBee wireless communication. The communication flow of each node in the ZigBee wireless communication network is as follows. After the initialization, CC2530 sends network request to server. If server permits the request, it becomes a communication node and works in static power down mode immediately[4]. Once the interrupt request is received, the node's mode converts to the fully 
functional status. Otherwise it continues to wait. The node in fully functional state receives analog signals collected by the sensor and completes A/D conversion. Then the digital signals are transmitted to the monitoring server by the ZigBee network. After the transmission, the node's mode returns to static power down mode. The ZigBee communication flow is shown in Fig.3.

CGI. CGI (Common Gateway Interface) provides an execution path of external program for web server, and this server technology establishes the interaction between browser and web server[5].

There are three CGI processes: login.cgi, data.cgi and command.cgi. They can realize user's authentication, data transmission and remote control respectively. Firstly, the browser sends the user's request to the web server. If web server answers the request, it will send a command to CGI programs by using STDIN method. After executing data.cgi, equipment information in the buffer can be obtained. Finally, the HTML files which contain the equipment information are sent to the web server by using STDOUT method. And then they are sent to the browser by the web server and displayed in the remote terminals. The command function flow chart is shown in Fig.4, and the data function flow chart is shown in Fig.5.

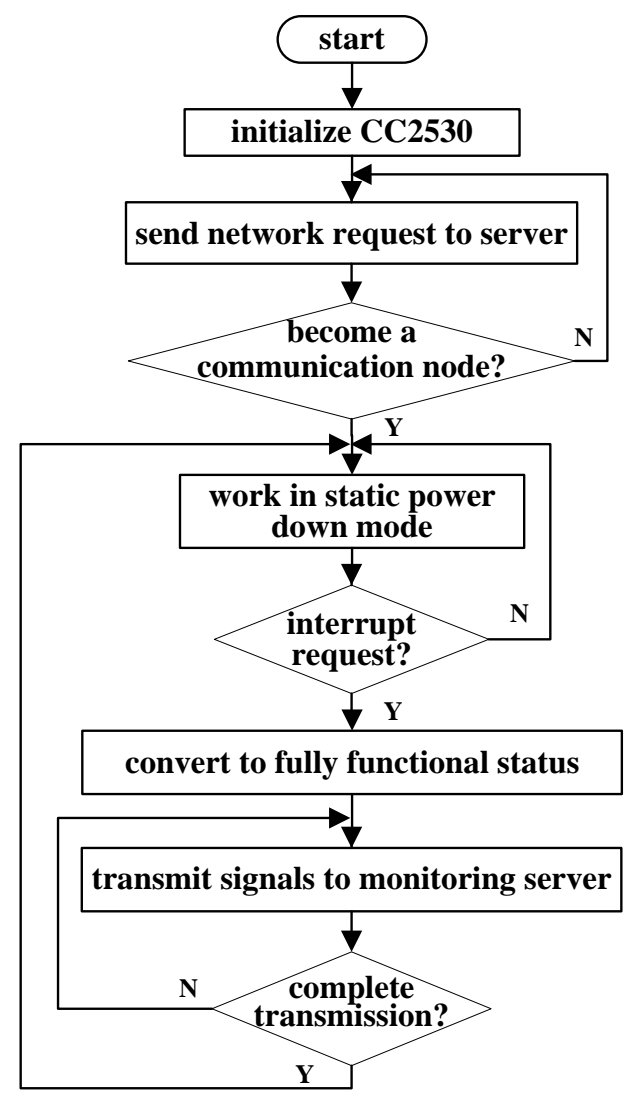

Fig.3. ZigBee wireless communication flow chart

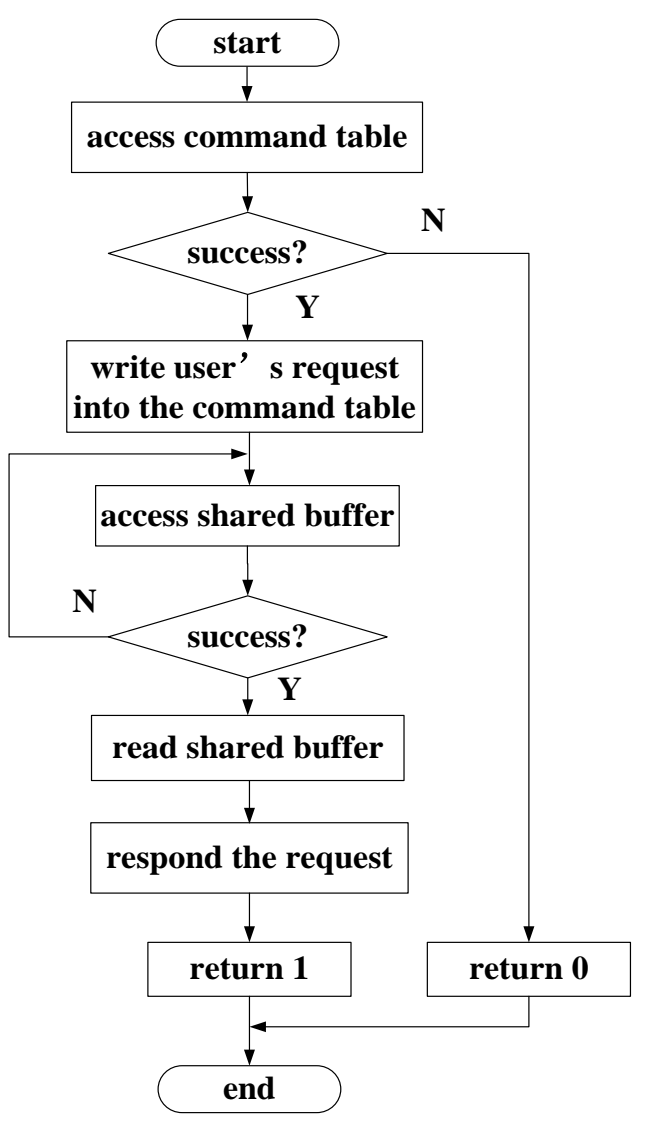

Fig.4. The command function flow chart

Socket Technology. Socket programs are designed to transmit data from the monitoring server to the FLASH monitoring module in client. UDP (User Data Protocol) is chosen to be the communication protocol. UDP is one of TCP/IP protocol. Before the transmission, client and server do not need to connect with each other. So the connection status is not required to be maintained. Then the server can transmit the same data to many clients simultaneously. And UDP requires less system resources than TCP (Transmission Control Protocol). The socket communication based on UDP is shown in Fig.6. 


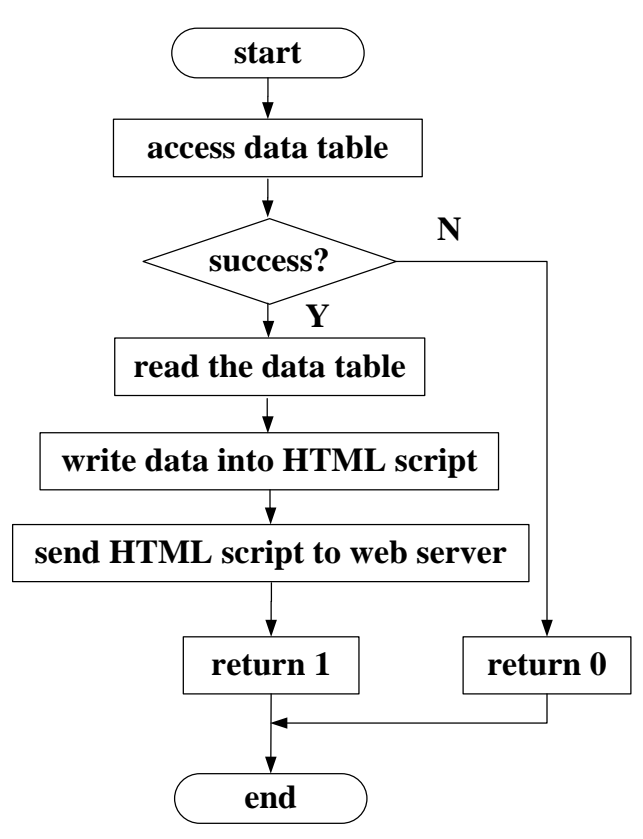

Fig.5. The data function flow chart

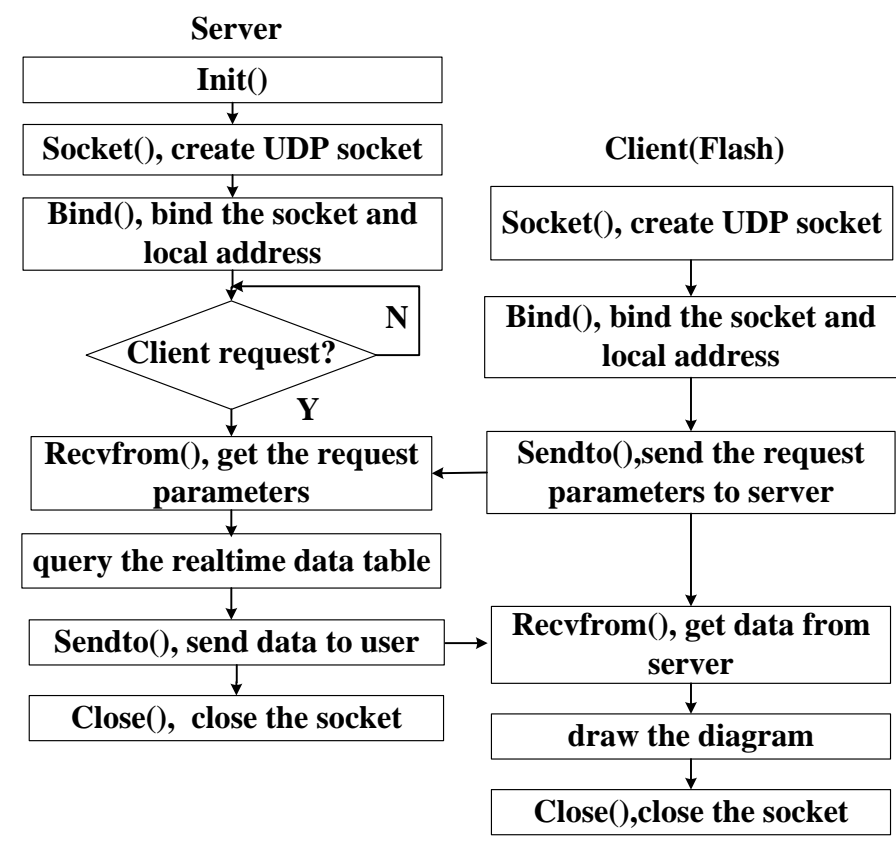

Fig.6. The socket communication based on UDP

FLASH Technology. HTML technology only provides page-based refresh method. When the page contains dynamic images, screen will flicker constantly. This is a serious matter. The stateless response mechanism and the stateless refresh mechanism of FLASH technology can solve this problem[6].

FLASH monitoring module sends request to the server per second automatically. FLASH plug-in calls the data acquisition procedures which get the equipment status by accessing SQLite database and then send the value to the FLASH module in a fixed string format. The two-dimensional curve is drawn in the client memory and then shown on the canvas without flicker. The key codes are shown as follows.

function paint(Graphics g)\{

Dimension $\mathrm{g}=$ getSize () ;

BufferedImage bimg;

bimg $=($ BufferedImage $)$ createImage (g.width, g.height $)$;

// Create the drawing buffer as large as the drawing area.

...// Draw the coordinate axis and coordinate.

bimg .createEmptyMovieClip("drawobj",1);

// Create a MovieClip dynamically.

drawobj.linestyle(1,0x111111,100);

// Set the thickness, color and transparency of the line.

loadVariables (“data.c",_root,"POST");

//Acquire data from the database and bind it to the _root.

drawobj.lineto(_root._x,_root._y);

//Draw the real-time curve in the client memory.

g.drawImage(bimg, 0,0 , null);

//Show the real-time graph on the canvas.

\}

\section{The Realization of Remote Monitoring}

When users access the home page in the server by browser, the HTML and FLASH programs embedded in the home page are started. The horizontal and vertical vibration, displacement, temperature, voltage of the equipment can be monitored. If users choose any one of the signals, it's 
dynamic oscillograms will be displayed. The oscillograms are put into the swf files and embedded into the home page. Users also can set the sample rate, channel number and trigger level. Test of remote monitoring is shown in Fig.7.

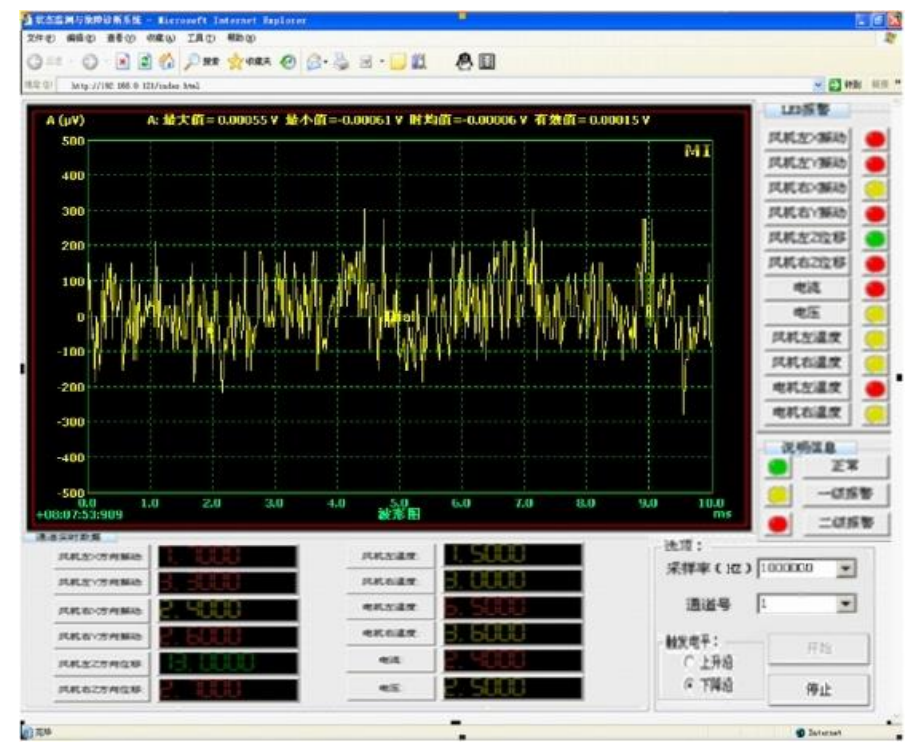

Fig.7. Test of remote monitoring

\section{Conclusion}

In the remote monitoring system, various real-time communication technologies are applied. Firstly, ZigBee technology is adopted to build a wireless communication network with various sensors. Secondly, an embedded ZigBee-Ethernet intelligent gateway is designed. And it realizes a seamless connection between the ZigBee wireless communication network and Internet. In addition, the remote communication is achieved via the CGI technology and the socket technology based on UDP. Finally, by means of powerful dynamic graphics of FLASH technology, the data refresh and dynamic oscillogram are realized creatively.

\section{References}

[1] X.Y. MAO, Application of Embedded System in the Internet of Things, Computer and Network, Vol. 41(10), pp.52-53, 2015.

[2] H.D. Zhang, L .Zhu. Internet of Things: Key technology, Architecture and Challenging Problems, 2011 IEEE International Conference on Computer Science and Automation Engineering, Vol. 04, pp.527-532, 2011.

[3] J.T.JIANG, Y.HAN, X.L. ZHANG, Design and Implementation of University Assets and Labs Integrated Management Platform Based on $\mathrm{B} / \mathrm{S}$ and $\mathrm{C} / \mathrm{S}$ Collaborative Services, Computer Applications and Software, Vol. 31(3), pp.47-50, 2014.

[4] D. XU, W.Y. CAO, Q. SUN, Design of Wireless Sensor Network Node for Environment Monitoring Based on CC2530, Journal of Computer Applications, Vol.33, pp.17-20, 2013.

[5] B.Z. Wang, C. Ma, Study and Implementation of the Embedded Dynamic Web Based on CGI Technology, Electronic Design Engineering, Vol.20(18), pp.161-163, 2012.

[6] X.B. SUN, R.H. WANG, P.J. ZHANG, Design of Remote Industry Control System Based on Flash, Electronic Design Engineering, Vol.20(9), pp.64-67, 2012. 